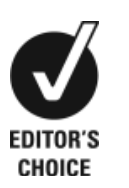

${ }^{1}$ Section of Oral and

Maxillofacial Oncology, Division

of Maxillofacial Diagnostic and

Surgical Sciences, Faculty of

Dental Science, Kyushu

University, Fukuoka, Japan

${ }^{2}$ Division of Nephrology and Rheumatology, Department of Internal Medicine, Faculty of Medicine, Fukuoka University,

Fukuoka, Japan

\section{Correspondence to}

Dr Masafumi Moriyama, Section of Oral and

Maxillofacial Oncology, Division of Maxillofacial Diagnostic and Surgical Sciences, Faculty of Dental Science, Kyushu

University, 3-1-1 Maidashi,

Higashi-ku, Fukuoka 812-8582,

Japan; moriyama@dent.

kyushu-u.ac.jp

Accepted 20 May 2012 Published Online First 29 June 2012

\title{
Interleukin-21 contributes to germinal centre formation and immunoglobulin G4 production in IgG4-related dacryoadenitis and sialoadenitis, so-called Mikulicz's disease
}

\author{
Takashi Maehara, ${ }^{1}$ Masafumi Moriyama, ${ }^{1}$ Hitoshi Nakashima, ${ }^{2}$ Katsuhisa Miyake, ${ }^{2}$ \\ Jun-Nosuke Hayashida, ${ }^{1}$ Akihiko Tanaka, ${ }^{1}$ Shouichi Shinozaki, ${ }^{1}$ Yoshiaki Kubo, ${ }^{1}$ \\ Seiji Nakamura ${ }^{1}$
}

\begin{abstract}
Objectives Interleukin (IL)-21 is mainly produced by CD4 T helper (Th) cells including Th2, Th17 and follicular helper $\mathrm{T}$ (Tfh) cells. Recent studies have reported that IL21 is involved in the formation of germinal centres (GCs) and class switching of lgG4. It has been suggested that IgG4-related dacryoadenitis and sialoadenitis (IgG4-DS), so-called Mikulicz's disease (MD), is distinct from Sjögren's syndrome (SS) and shows a high frequency of GC formation in salivary glands. In this study the expression of IL-21 in lgG4-DS and SS patients was examined.
\end{abstract}

Methods Twelve patients with IgG4-DS, 15 with SS and 15 healthy subjects were screened for (1) ectopic GC formation in formalin-fixed labial salivary gland (LSG) biopsy samples; (2) expression of IL-21, Th2-, Th17- and Tfh-related molecules (cytokines, chemokine receptors and transcription factors) in LSGs; (3) relationship between $\operatorname{lgG} 4 / \mathrm{lgG}$ ratio and mRNA expression of IL-21 in LSGs.

Results mRNA expression of IL-21 and Bcl-6 in LSGs from patients with lgG4-DS was significantly higher than in patients with SS and controls. IL-21 and CXCR5 were detected by immunohistochemistry in or around GC in patients with SS and those with IgG4-DS. IL-21 was detected in infiltrating lymphocytes outside GC only in patients with IgG4-DS. Expression of IL-21 was consistent with that of Th2-related molecules while IL-17 was rarely seen in IgG4-DS. Furthermore, the expression of IL-21 in LSGs was correlated with the number of GC formations and the $\lg \mathrm{G} 4 / \mathrm{lgG}$ ratio in patients with $\lg \mathrm{G} 4-\mathrm{DS}$.

Conclusions These results suggest that overexpression of IL-21 by Th2 cells might play a key role in GC formation and lgG4 production in lgG4-DS.

Mikulicz's disease (MD) has been considered to be a subtype of Sjögren's syndrome (SS) based on the histopathological similarities between the two diseases. 12 Yamamoto et al reported that patients with $\mathrm{MD}$ had elevated serum IgG4 and infiltration of IgG4-positive plasma cells in the gland tissues. These findings have also been found in autoimmune pancreatitis, ${ }^{3}$ sclerosing cholangitis, ${ }^{4}$ tubulointerstitial nephritis, ${ }^{5}$ interstitial pneumonia, ${ }^{6}$ Ridel's thyroiditis ${ }^{7}$ and Küttner's tumour. ${ }^{8}$ These diseases are thus now referred to as
'IgG4-related disease' (IgG4-RD). ${ }^{9} 10$ We recently described the concept of IgG4-RD and provided up-to-date information on this emerging disease entity. ${ }^{11}$ Recent studies have referred to $\mathrm{MD}$ as 'IgG4-related dacryoadenitis and sialoadenitis' (IgG4-DS). ${ }^{11-14}$ Histologically, ectopic germinal centres (GCs) can frequently occur in the salivary glands in IgG4-DS. It is generally accepted that interleukin (IL)-21 plays a crucial role in the development of GC formation and is mainly produced by CD4 T cells (ie, T helper (Th) 2 cells, ${ }^{15}$ Th17 cells $^{16}$ and $\mathrm{T}$ follicular helper (Tfh) cells ${ }^{17}{ }^{18}$ ). In particular, IL-21 production by Tfh cells, which are characterised by expression of CXC chemokine receptor 5 (CXCR5), help GC formation. ${ }^{18}$ High levels of IL-21 receptors are present at the surface of most B cells. ${ }^{17}$

We previously reported that peripheral CD4 T cells from patients with IgG4-DS have deviation of Th1/Th2 balance to Th2 and elevated expression of Th2-type cytokines, ${ }^{19}$ suggesting that IgG4-DS has a Th2-predominant phenotype. ${ }^{20}$ We also demonstrated a close association of IL-4 and IL-10 expression with IgG4 production in the labial salivary glands (LSGs) of patients with IgG4-DS. ${ }^{21} 22$ Furthermore, recent studies in humans and mice reported that class switching of IgG4 is caused by co-stimulation with IL-4 and IL-21. ${ }^{23-25}$

However, to our knowledge, no published reports have investigated the involvement of IL-21 expression in class switching of IgG4 in the salivary glands of patients with IgG4-DS. We therefore examined infiltrating lymphocytes expressing IL-21 in LSGs from patients with IgG4-DS to clarify the contribution of IL-21 to the pathogenesis of IgG4-DS.

\section{METHODS \\ Patients}

Twelve patients with IgG4-DS (nine women and three men, mean \pm SD age $61.8 \pm 8.1$ years) and 15 patients with primary SS (14 women and one man, mean $\pm S D$ age $61.4 \pm 9.8$ years) who were referred to the Department of Oral and Maxillofacial Surgery, Kyushu University Hospital between 2007 and 2011 were included in the study. Medical records were retrospectively 
reviewed after the diagnosis. IgG4-related MD (IgG4-DS) was diagnosed according to the following criteria: ${ }^{211}$ (1) persistent symmetrical swelling of at least two pairs of lachrymal and major salivary glands for at least 3 months; (2) raised serum levels of IgG4 (>135 mg/dl); and (3) infiltration of IgG4-positive plasma cells in the tissue (IgG4 + cells/IgG + cells $>50 \%$ ). The clinical and serological characteristics of the 12 patients with IgG4-DS are summarised in table 1.

SS was diagnosed according to the Research Committee on SS of the Ministry of Health and Welfare of the Japanese Government (1999) and the American-European Consensus Group criteria for SS. ${ }^{26}$ Each patient showed objective evidence of salivary gland involvement based on the presence of subjective xerostomia and a decreased salivary flow rate, abnormal findings on parotid sialography and focal lymphocytic infiltrates in the LSGs. There was no documented history of treatment with steroids, HIV, HTLV-1, hepatitis B virus or hepatitis $C$ virus infection, sarcoidosis or any other immunodepressants in any of the patients. None of the patients had evidence of malignant lymphoma at the time of the study. The prevalences of anti-SS-A/Ro, anti-SS-B/La and antinuclear antibodies were $72.6 \%, 33.2 \%$ and $71.6 \%$, respectively. LSG biopsies were performed as described by Greenspan et al. ${ }^{27}$ Fifteen patients with mucocoeles (11 women and four men, mean \pm SD age 58.4 \pm 16.3 years) with no clinical or laboratory evidence of systemic autoimmune disease were chosen as a control group. All control LSGs were histologically normal. The clinical and serological characteristics of the 15 patients with SS are summarised in table 2 .

The study was approved by the ethics committee of Kyushu University, Japan and informed consent was obtained from all the patients and healthy controls included in the study.

\section{Histology}

Formalin-fixed and paraffin-embedded $4 \mu \mathrm{m}$ sections of LSG specimens were prepared and stained with haematoxylin and eosin (HE) for conventional histological examination. The degree of lymphocytic infiltration in the specimens was judged by focus scoring. ${ }^{21} 2728$ One standardised score was the number of focal inflammatory cell aggregates containing $\geq 50$ mononuclear cells in each $4 \mathrm{~mm}^{2}$ area of salivary gland tissue. $^{29}$

\section{Evaluation of ectopic GCs}

Ectopic GCs have been previously defined by the presence of $\mathrm{B}$ and $\mathrm{T}$ cell follicles, follicular dendritic cell networks and high densities of endothelial venules and proliferating cells. ${ }^{30} 31$ In this study, HE-stained LSG tissue sections from 20 patients with IgG4-DS and 66 patients with primary SS who were referred to the Department of Oral and Maxillofacial Surgery, Kyushu University Hospital between 1993 and 2011 were evaluated for the frequency, number and size of ectopic GC formations. The numbers of GC were counted in $4 \mathrm{~mm}^{2}$ sections from five different areas. Ectopic GCs were defined by HE staining as well-circumscribed chronic inflammatory cell infiltrates consisting of at least 50 mononuclear cells presenting with a densely-packed dark zone and a less densely-packed light zone. Only a few of the structures defined in this way corresponded to real GCs.

\section{RNA extraction and complementary DNA (cDNA) synthesis}

Total RNA was prepared from the whole LSGs using the acidified guanidinium-phenol-chloroform method, as previously described. ${ }^{21} 32$ Three micrograms of the total RNA preparation was then used for the synthesis of cDNA. Briefly, RNA was incubated for $1 \mathrm{~h}$ at $42^{\circ} \mathrm{C}$ with $20 \mathrm{U}$ RNasin ribonuclease inhibitor (Promega, Madison, Wisconsin, USA), $0.5 \mathrm{mg}$ oligo-(dT) 1218 (Pharmacia, Uppsala, Sweden), $0.5 \mathrm{mM}$ of each dNTP (Pharmacia), $10 \mathrm{mM}$ dithiothreitol and $100 \mathrm{U}$ RNase H reverse transcriptase (Life Technologies, Gaithersburg, Maryland, USA).

\section{Quantitative estimation of mRNA by real-time polymerase chain reaction (PCR)}

Quantitative cDNA amplification from whole LSGs and microdissected samples was performed according to the manufacturer's instructions and previous reports. ${ }^{21} 32$ cDNAs of the cytokines and transcription factors were analysed by real-time PCR using Light Cycler Fast Start DNA Master SYBR Green 1 (Roche Diagnostics, Mannheim, Germany) in a Light Cycler

Table 1 Clinical and serological findings of 12 patients with (lgG4-DS)

\begin{tabular}{|c|c|c|c|c|c|c|c|c|c|c|c|c|c|}
\hline \multicolumn{4}{|c|}{ Patients } & \multirow{3}{*}{$\begin{array}{l}\text { Lymphocytic } \\
\text { infiltration }^{\dagger}\end{array}$} & \multirow{2}{*}{\multicolumn{5}{|c|}{$\frac{\text { Clinical findings }}{\text { Swelling glands }}$}} & \multicolumn{4}{|c|}{ Serological findings } \\
\hline \multirow[b]{2}{*}{ No. } & \multirow[b]{2}{*}{ Age } & \multirow[b]{2}{*}{ Sex } & \multirow{2}{*}{$\begin{array}{l}\text { Disease } \\
\text { duration }\end{array}$} & & & & & & & \multirow[b]{2}{*}{ SS-A/Ro } & \multirow[b]{2}{*}{ SS-B/La } & \multirow[b]{2}{*}{$\operatorname{lgG}(872 \sim 1815 \mathrm{mg} / \mathrm{dl})$} & \multirow{2}{*}{$\begin{array}{l}\operatorname{lgG} 4(95 \sim 105 \\
\mathrm{mg} / \mathrm{dl})\end{array}$} \\
\hline & & & & & LG & PG & SMG & SLG & Complications & & & & \\
\hline 1 & 76 & $\mathrm{~F}$ & $4 \mathrm{M}$ & 11 & & & & & DM & - & - & 2381 & 823 \\
\hline 2 & 70 & $\mathrm{~F}$ & $5 Y$ & 12 & & & & & - & - & - & 4410 & 1930 \\
\hline 3 & 60 & $\mathrm{~F}$ & $1 Y$ & 11 & & & & & AIP, SC & - & - & 1614 & 490 \\
\hline 4 & 64 & $\mathrm{~F}$ & $1 Y$ & 10 & & & & & AIP & - & - & 2430 & 896 \\
\hline 5 & 79 & $\mathrm{~F}$ & $10 \mathrm{M}$ & 11 & & & & & AIP, SC & - & - & 2585 & 456 \\
\hline 6 & 61 & $\mathrm{~F}$ & $3 \mathrm{M}$ & 9 & & & & & AIP, SC & - & - & 2891 & 1080 \\
\hline 7 & 57 & $\mathrm{~F}$ & $6 \mathrm{M}$ & 10 & & & & & AIP, SC & - & - & 1842 & 748 \\
\hline 8 & 65 & $\mathrm{M}$ & $5 \mathrm{M}$ & 10 & & & & & Hydronephrosis & - & - & 3142 & 1700 \\
\hline 9 & 31 & $\mathrm{~F}$ & $1.5 Y$ & 12 & & & & $\Omega$ & AIP, DM & - & - & 2055 & ND \\
\hline 10 & 70 & $\mathrm{M}$ & $1 \mathrm{M}$ & 11 & & & & & Asthma & - & - & 2010 & ND \\
\hline 11 & 61 & $\mathrm{M}$ & $3 \mathrm{M}$ & 10 & & & & 0 & $\begin{array}{l}\text { Pulmonary } \\
\text { nodules }\end{array}$ & - & - & 7603 & 2290 \\
\hline 12 & 48 & $\mathrm{~F}$ & $3 \mathrm{M}$ & 8 & 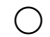 & & & & Asthma & - & - & 2401 & ND \\
\hline
\end{tabular}

AIP, autoimmune pancreatitis; LG, lachrymal gland; LSG, labial salivary gland; -, negative; ND, not done; PG, parotid gland; SC, sclerosing cholangitis; SLG, sublingual gland; SMG, submandibular gland. 
Table 2 Clinical and serological findings of 15 patients with primary Sjögren's syndrome (SS)

\begin{tabular}{|c|c|c|c|c|c|c|c|c|c|c|c|}
\hline \multirow[b]{3}{*}{ No. } & \multirow[b]{3}{*}{ Age } & \multirow[b]{3}{*}{ Sex } & \multirow{3}{*}{$\begin{array}{l}\text { Lymphocytic } \\
\text { infiltration* }\end{array}$} & \multicolumn{5}{|l|}{ Autoantibody } & \multicolumn{3}{|l|}{ Immunoglobulin } \\
\hline & & & & RF & ANA & DNA & SS-A/Ro & SS-B/La & $\lg \mathbf{g}$ & $\lg A$ & $\lg M$ \\
\hline & & & & $(20 \sim \geqq \mathrm{U} / \mathrm{ml})$ & (titer) & $(10 \sim>\mathrm{U} / \mathrm{ml})$ & $(10 \sim>)$ & $(15 \sim>)$ & (872 1815 mg/dl) & $(95 \sim 405 \mathrm{mg} / \mathrm{dl})$ & $(59 \sim 269 \mathrm{mg} / \mathrm{dl})$ \\
\hline 2 & 44 & $\mathrm{~F}$ & 9 & $<20$ & Negative & Negative & 104 & 29 & 1517 & 193 & 174 \\
\hline 3 & 65 & $\mathrm{~F}$ & 9 & ND & 80 & ND & 64 & $<15$ & ND & ND & ND \\
\hline 4 & 52 & $\mathrm{~F}$ & 11 & 40 & $>1280$ & $<5$ & $>256$ & 32 & 2206 & 270 & 107 \\
\hline 7 & 53 & $\mathrm{~F}$ & 11 & ND & ND & ND & $<10$ & $<15$ & ND & ND & ND \\
\hline 8 & 59 & $\mathrm{~F}$ & 12 & ND & ND & ND & $>256$ & $<15$ & ND & ND & ND \\
\hline 9 & 89 & $\mathrm{M}$ & 8 & $4<$ & Negative & ND & 44.6 & $<15$ & 2239 & 461 & 54 \\
\hline 10 & 29 & $\mathrm{~F}$ & 9 & 40 & $>1280$ & ND & $>256$ & $<15$ & ND & ND & ND \\
\hline 11 & 92 & $\mathrm{~F}$ & 7 & 29 & 320 & ND & 64 & 2 & 1565 & 581 & 103 \\
\hline 15 & & & & & & & & & & & \\
\hline
\end{tabular}

${ }^{*}$ The degree of lymphocytic infiltration in the LSGs was graded from 1 to 12 by focus scoring.

Real-time PCR instrument (V.3.5; Roche Diagnostics). The cytokines, chemokine receptors and transcription factors examined in the current study were IL-4, IL-17, IL-21, CC chemokine receptor 4 (CCR4), CXCR5, Bcl-6 and retinoic acid-related orphan receptor $\mathrm{C} 2$ (RORC2). ${ }^{33}$ The lymphocyte markers examined were IgG and IgG4. The primer sequences used were as follows: $\beta$-actin, forward $5^{\prime}$-GCA AAG ACC TGT ACG CCA AC-3', reverse 5'-CTA GAA GCA TTT GCG GTG GA-3', $258 \mathrm{bp}$; IL-4, forward 5'-GCA GTT CCA CAG GCA CAA-3', reverse 5'-CTC TGG TTG GCT TCC TTC AC-3', 108 bp; IL-17, forward 5'-CCC CAG TTG ATT GGA AGA AA-3', reverse 5'-AGA TTC CAA GGT GAG GTG GA-3', 252 bp; IL-21, forward 5'-CCA CAA ATC AAG CTC CCA AG-3', reverse 5'-CAG GGA CCA AGT CAT TCA CA-3', 258 bp; CCR4, forward 5'-GTG CTC TGC CAA TAC TGT GG-3', reverse 5'-CTT CCT CCT GAC ACT GGC TC-3', 214 bp; CXCR5, forward 5'-GGT CTT CAT CTT GCC CTT TG-3', reverse 5'-ATG CGT TTC TGC TTG GTT CT-3', 340 bp; Bcl-6, forward 5'-GAA GCC CTA CAA ATG CGA AA-3', reverse 5'-TGA CGG AAA TGC AGG TTA CA-3', 210 bp; RORC2, forward 5'-GGG TAC AAT GAA GGC CAA GA-3', reverse 5'-AGC TGT GGC CTC AAG GAT AA-3', 211 bp; IgG, forward 5'-CAA GTG CAA GGT CTC CAA CA-3', reverse 5'-TGG TTC TTG GTC AGC TCA TC-3', $129 \mathrm{bp}$; IgG4, forward 5'-TGA CGG TGT CGT GGA ACT-3', reverse 5'-ACG TTG CAG GTG TAG GTC T-3', 145 bp.

In order to provide a meaningful comparison between different individuals or samples, the amounts of the PCR products were calculated relative to the amounts of $\beta$-actin PCR products (for the standardisation of total cellular mRNA) in each sample, as previously described. ${ }^{21} 34$

\section{Immunohistochemical analysis}

For immunohistochemical analysis, $4 \mu \mathrm{m}$ formalin-fixed and paraffin-embedded sections were prepared and stained using a conventional avidin-biotin complex technique, as described previously. ${ }^{21} 35$ The polyclonal antibodies used to analyse the cytokines were anti-IL-4 (clone: ab9622; Abcam, Cambridge, UK), anti-IL-17 (clone: sc-7927; Santa Cruz Biotechnology,
Santa Cruz, California, USA), anti-IL-21 (clone: LS-C401; LifeSpan BioScience, LSBio, Seattle, Washington, USA) and anti-c-Maf (clone: ab77071; Abcam). SS1 (anti-sheep erythrocyte IgG2a), NS8.1 (anti-sheep erythrocyte IgG2b) and NS4.1 (anti-sheep erythrocyte IgM) were used as control rabbit polyclonal antibodies. The mouse monoclonal antibodies used to analyse the transcription factors were anti-Bcl-6 (clone: ab9479; Abcam), anti-CCR4 (MAB1567; R\&D Systems) and anti-CXCR5 (clone: ab89259; Abcam). HDP-1 (anti-DNP IgG1) was used as a control mouse monoclonal antibody. The sections were sequentially incubated with primary antibodies, biotinylated anti-mouse IgG secondary antibodies (Vector Laboratories, Burlingame, California, USA), avidin-biotinhorseradish peroxidase complex (Vector Laboratories) and 3,3'-diaminobenzidine (Vector Laboratories). Mayer's haematoxylin was used for counterstaining. Photomicrographs were obtained using a light microscope equipped with a digital camera (CoolSNAP; Photometrics, Tucson, Arizona, USA). Stained IgG4-positive and IgG-positive cells were counted in $1 \mathrm{~mm}^{2} \mathrm{sec}-$ tions from five different areas and the ratio of IgG4-positive cells to IgG-positive cells was calculated.

\section{Statistical analysis}

The significance of differences between groups was determined using $\chi^{2}$ tests, Student $t$ tests, Mann-Whitney $U$ tests and Spearman rank correlations. All statistical analyses were performed using JMP software (V.8; SAS Institute, Japan). A p value $<0.05$ was considered statistically significant.

\section{RESULTS}

\section{Formation of ectopic GCs in LSGs from patients with SS and IgG4-DS}

SS was characterised by periductal lymphocytic infiltration with atrophy or severe destruction of the acini, while IgG4-DS showed non-periductal lymphocytic infiltration with hyperplastic GCs and mild destruction of the acini (figure $1 \mathrm{~A}$ ). Fifteen of 66 patients with SS (23\%) and 12 of 20 patients with IgG4-DS (60\%) showed ectopic GC formation in the formalin-fixed tissue. Patients with IgG4-DS showed 
A
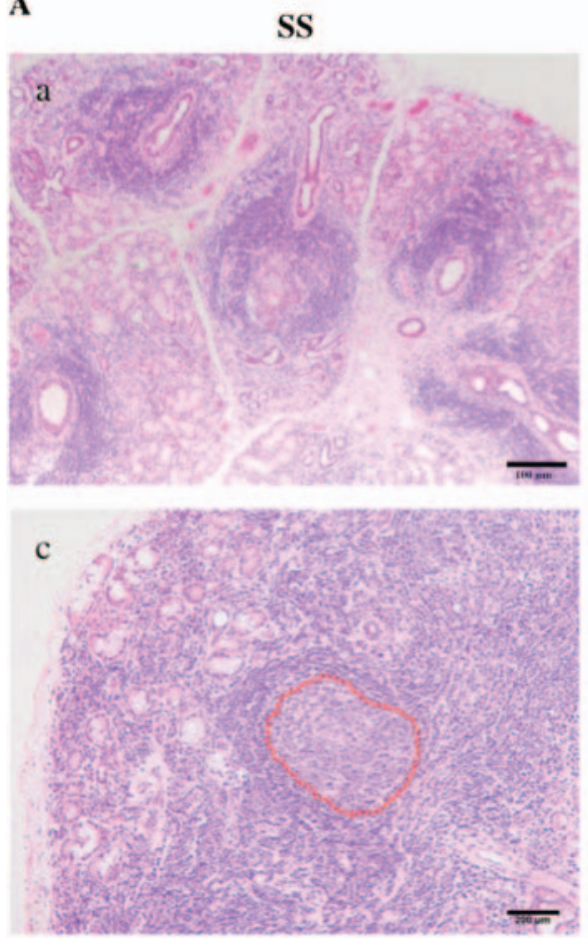
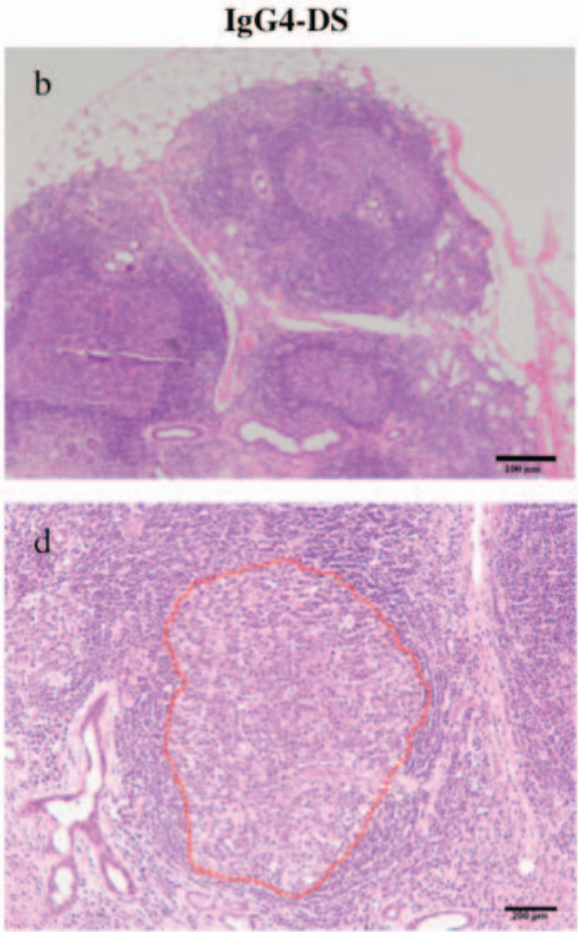

B

\begin{tabular}{cccc} 
B & SS & IgG4-DS & $P$ value \\
\hline Frequency & $15 / 66(22.7 \%)$ & $12 / 20(60 \%)$ & $P<0.05 \dagger$ \\
Number $(/ \mathrm{HPF})$ & $0.1 \pm 0.3$ & $2.5 \pm 2.6$ & $P<0.05 \div ;$ \\
Size $\left(\times 10^{3} \mu \mathrm{m}^{2}\right)$ & $31.2 \pm 21.9$ & $68.1 \pm 29.4$ & $P<0.05 \div ;$
\end{tabular}

Figure 1 (A) Germinal centre (GC) formation in labial salivary gland (LSG) sections from representative patients with Sjögren's syndrome (SS) and IgG4-related dacryoadenitis and sialoadenitis (IgG4-DS). The degrees of lymphocytic infiltration in LSGs from patients with SS and IgG4-DS had focus scores of 11. IgG4-DS showed lymphocytic infiltration with extensive ectopic GC formation compared with SS (a, b). The area enclosed by the red line shows the ectopic GCs in the LSGs from patients with SS and IgG4-DS (c, d). Scale bars, 100 and $200 \mu \mathrm{m}$. (B) Difference in ectopic GC formation between patients with SS and IgG4-DS. TSignificance of the difference between the two groups was determined by $\chi^{2}$ tests. t†Significance of the difference between the two groups was determined by the Student $t$ test. HPF, high-power field.

significantly higher frequency, higher number and larger size of these structures compared with those from patients with SS (figure 1B). The characteristics of the 15 patients with SS and 12 patients with IgG4-DS who were found to have these structures are summarised in table $1 \mathrm{~A}, \mathrm{~B}$, respectively. The patients with SS and IgG4-DS all had strong lymphocyctic infiltration and the mean \pm SD focus scores were $9.27 \pm 1.53$ and $10.4 \pm 1.16$, respectively. Focus scores between the two groups were not significantly different.

\section{Expression of IL-21 and Tfh-related molecules (CXCR5 and Bcl-6) in LSGs}

The mRNA expression levels of IL-21, CXCR5 and Bcl-6 in LSGs from SS and IgG4-DS patients were significantly higher than those in controls (figure $2 \mathrm{~A}$ ). In addition, the mRNA expression levels of IL-21 and Bcl-6 in the LSGs from patients with IgG4-DS were higher than those from patients with SS. Representative histological findings in the LSG specimens from patients with SS and IgG4-DS and from controls are shown in figure $2 \mathrm{Ba}-\mathrm{c}$. Patients with $\mathrm{SS}$ and IgG4-DS showed GC formation. The specimens were immunohistochemically examined to evaluate the distribution of the cytokines, chemokine receptors and transcription factors in the LSGs from patients with SS and IgG4-DS (figure 2B). Expression of IL-21, CXCR5 and Bcl-6 was detected in ectopic GCs from patients with SS and IgG4-DS (figure $2 \mathrm{Be}, \mathrm{f}, \mathrm{h}, \mathrm{i}, \mathrm{k}, \mathrm{l}$ ). Interestingly, the expression of IL-21 and Bcl-6 was also detected in infiltrating lymphocytes outside the ectopic GCs only in patients with IgG4-DS (figure 2Bf,1). Expression of IL-21 was slightly detected in ductal epithelial cells in the LSGs from patients with SS and controls (figure $2 \mathrm{Bd}, \mathrm{e}$ ). None of the LSGs from controls exhibited CXCR5 or Bcl-6 expression (figure $2 \mathrm{Bg}, \mathrm{j}$ ).

\section{Expression of Th2-related molecules (IL-4, CCR4 and C-Maf) and Th17-related molecules (IL-17 and RORC2) in LSGs}

As shown in figure 3A, mRNA expression levels of IL-4, CCR4, IL-17 and RORC2 in LSGs from patients with SS were significantly higher than in controls while mRNA expression levels of IL-4 and CCR4 were significantly higher in the LSGs from patients with IgG4-DS than in controls. Furthermore, the mRNA expression level of IL-4 was significantly higher in 
$\mathbf{A}$

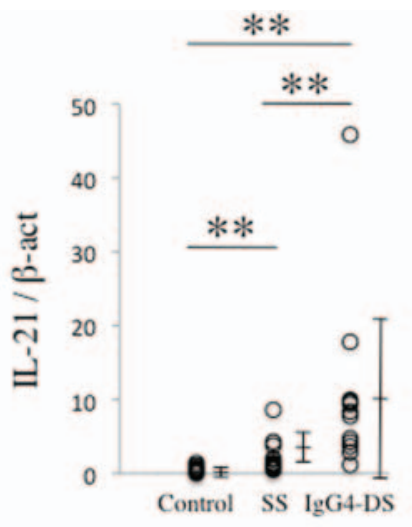

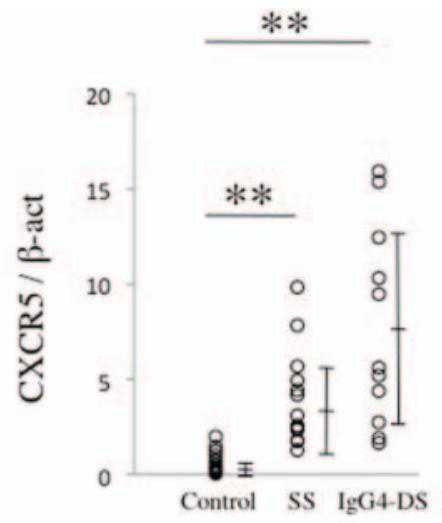

SS
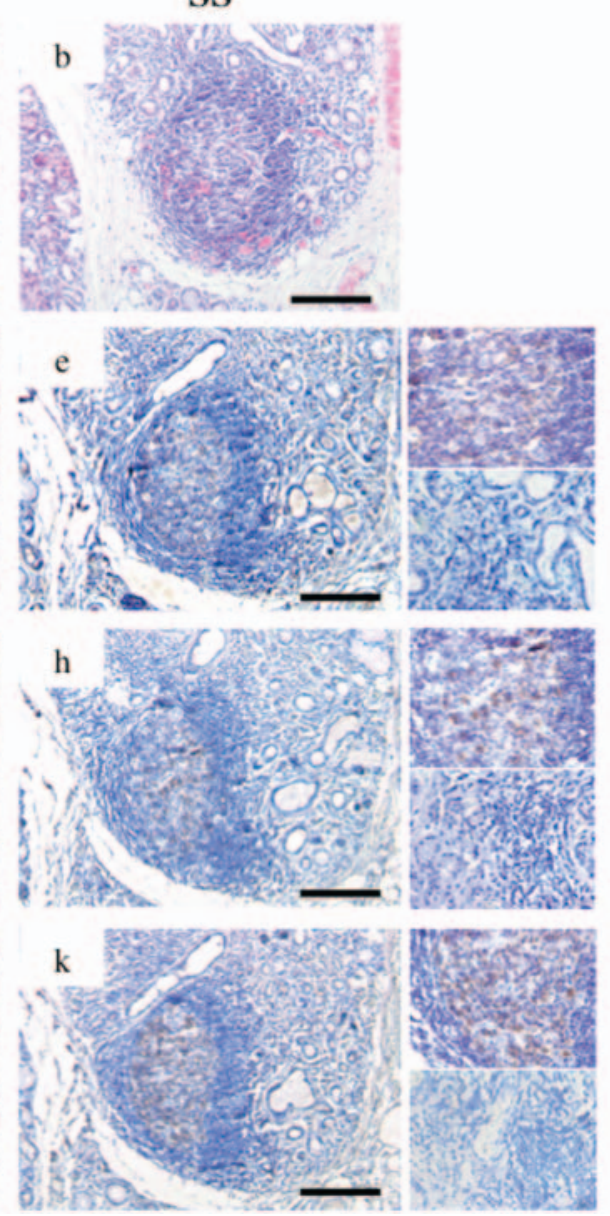

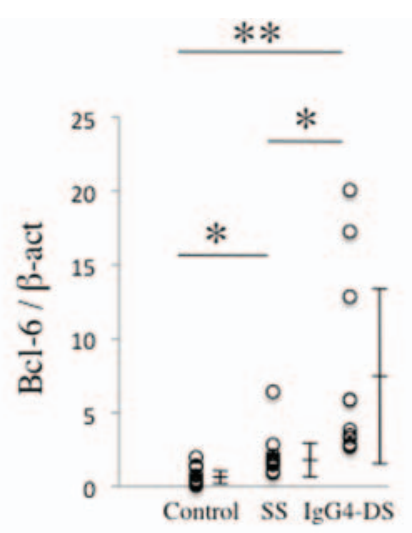

IgG4-DS
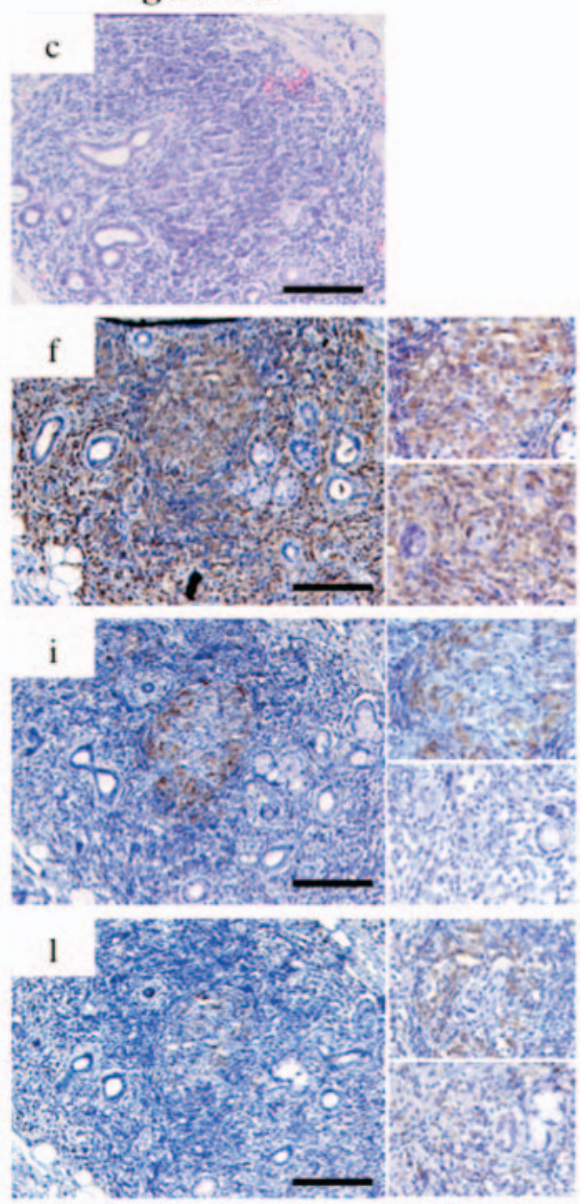

Figure 2 (A) mRNA expression levels of interleukin 21 (IL-21) in the labial salivary glands (LSGs) from patients with lgG4-related dacryoadenitis and sialoadenitis (IgG4-DS) were significantly higher than in those from patients with Sjögren's syndrome (SS) and controls. The mRNA expression levels of cytokine, chemokine receptor and transcription factor were examined in the LSGs from controls $(n=15)$, patients with SS $(n=15)$ and patients with IgG4-DS ( $n=12)$. IL-4, CXCR5 and Bcl-6 were quantitatively estimated as described in the Methods section. Bars represent means and SDs. Significant differences between groups were determined by the Mann-Whitney U test $\left({ }^{*} p<0.05,{ }^{*} p<0.01\right)$. (B) IL-21 was strongly expressed in whole LSGs only from patients with IgG4-DS. Staining with haematoxylin and eosin (HE) (a-c) and anti-IL-21 (d-f), anti-CXCR5 ( $\mathrm{g}-\mathrm{i})$ and anti-Bcl-6 ( $\mathrm{j}-1)$ monoclonal antibodies in the LSGs from a representative control, SS patient and IgG4-DS patient (brown). Counterstaining with Mayer's haematoxylin was performed subsequently (blue). The higher magnifications $(e, f, h, i, k, l)$ are displayed at the upper right (in the ectopic germinal centre) and the lower right (outside the ectopic ectopic centre). Scale bars, $100 \mu \mathrm{m}$.

LSGs from patients with IgG4-DS than in patients with SS. The specimens were immunohistochemically examined to evaluate the distribution of these molecules in the LSGs from patients with SS and IgG4-DS and controls (figure 3B). Expression of IL-4, CCR4 and c-Maf was prominently detected in the whole LSGs from patients with IgG4-DS compared with those from patients with SS (figure 3Bb,c,e,f, $h, i, k, 1)$. The expression of IL-17 was slightly detected in the ectopic GCs from patients with SS but rarely in the whole LSGs from patients with IgG4-DS or controls (figure 3Bj,k,l). None of the control LSGs exhibited IL-4, CCR4 or c-Maf expression (figure $3 \mathrm{Ba}, \mathrm{d}, \mathrm{g}$ ). 
A

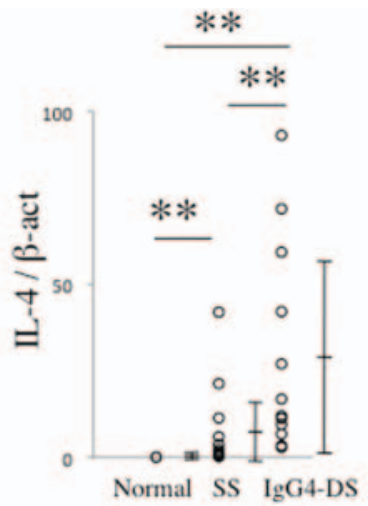

Control

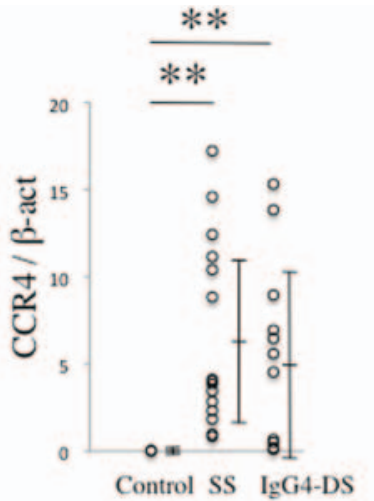

SS
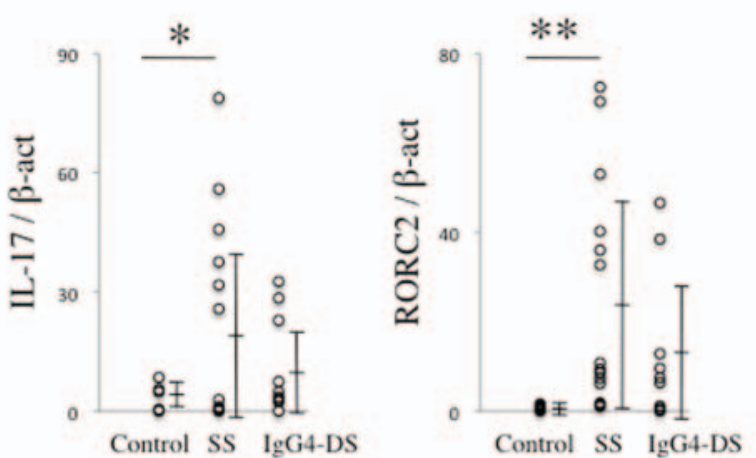

IgG4-DS

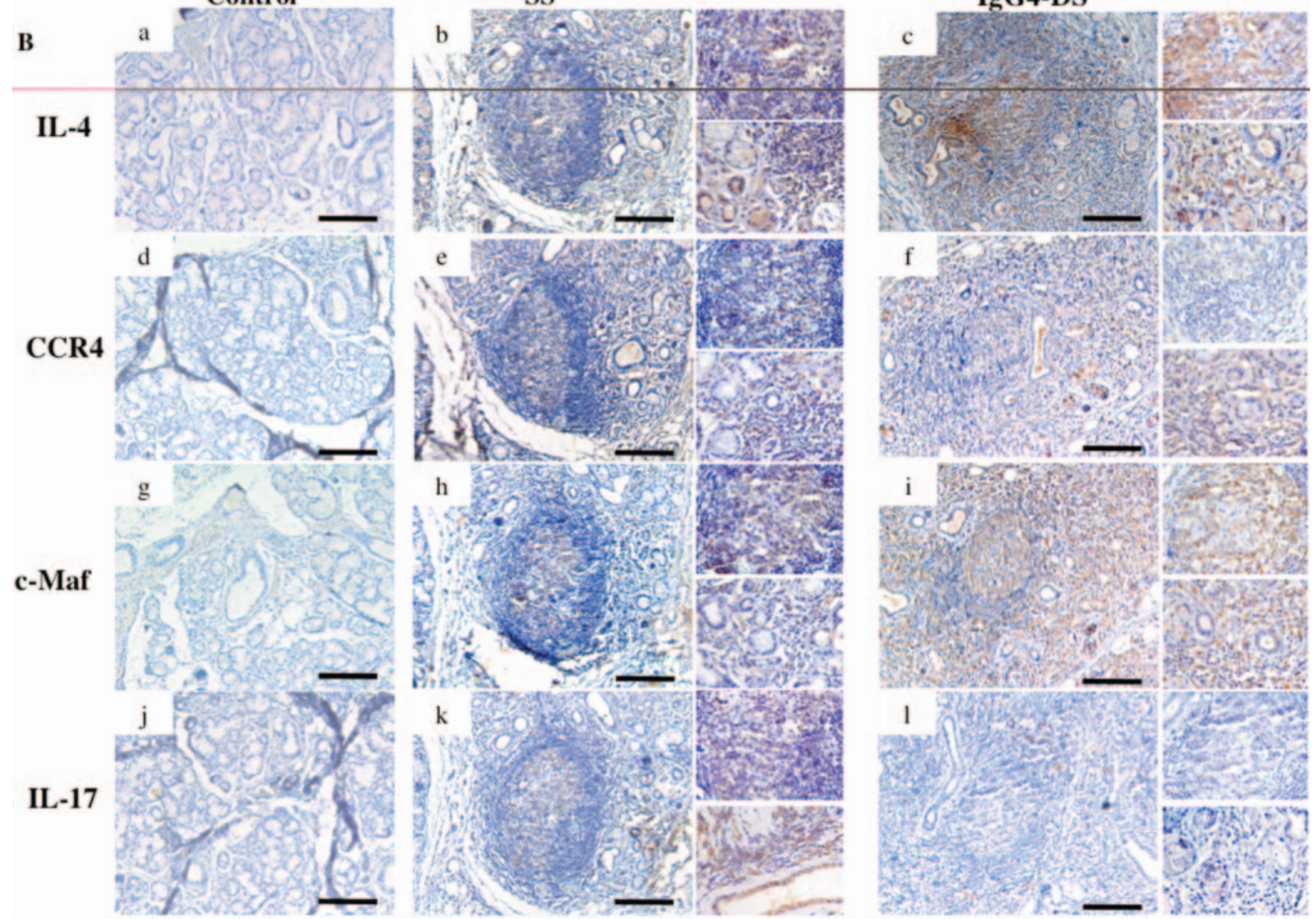

Figure 3 (A) mRNA expression levels of interleukin 4 (IL-4) in the labial salivary glands (LSGs) of patients with lgG4-related dacryoadenitis and sialoadenitis (IgG4-DS) were significantly higher than in those from patients with Sjögren's syndrome (SS). The mRNA expression levels of cytokine, chemokine receptor and transcription factor were examined in the LSGs from controls $(n=15)$, patients with SS $(n=15)$ and patients with lgG4-DS $(n=12)$. IL-4, CCR4, IL-17 and RORC2 were quantitatively estimated as described in the Methods section. Bars represent means and SDs. Significant differences between groups were determined by the Mann-Whitney $U$ test $\left({ }^{*} p<0.05,{ }^{* *} p<0.01\right)$. (B) IL-4, CCR4 and c-Maf were strongly expressed in whole LSGs from patients with IgG4-DS but not in patients with SS. Immunostaining with anti-IL-4 (a-c), anti-CCR4 (d-f), anti-c-Maf $(\mathrm{g}-\mathrm{i})$ and anti-IL-17 (j-I) monoclonal and polyclonal antibodies in the LSGs from a representative control, SS patient and IgG4-DS patient (brown). Counterstaining with Mayer's haematoxylin was performed subsequently (blue). The higher magnifications $(b, c, e, f, h, i, k, I)$ are displayed at the upper right (in the ectopic germinal centre) and the lower right (outside the ectopic germinal centre). Scale bars, $100 \mu \mathrm{m}$.

\section{Relationship between GC formation and expression of IL-21 in LSGs}

The relationship between the number of GC formations and levels of IL-21 mRNA expression in LSGs was examined. mRNA expression of IL-21 was positively correlated with the number of GC formations in LSGs from patients with IgG4-DS but not in those from patients with SS (figure 4).

\section{Relationship between IgG4 production and expression of IL-21 in LSGs}

The relationships between IgG4 production and levels of IL-21 mRNA expression in LSGs were examined. mRNA expression of IL-21 was positively correlated with the IgG4/IgG ratio in LSGs from patients with IgG4-DS but not with that in patients with SS (figure 5A). Patients with IgG4-DS showed 


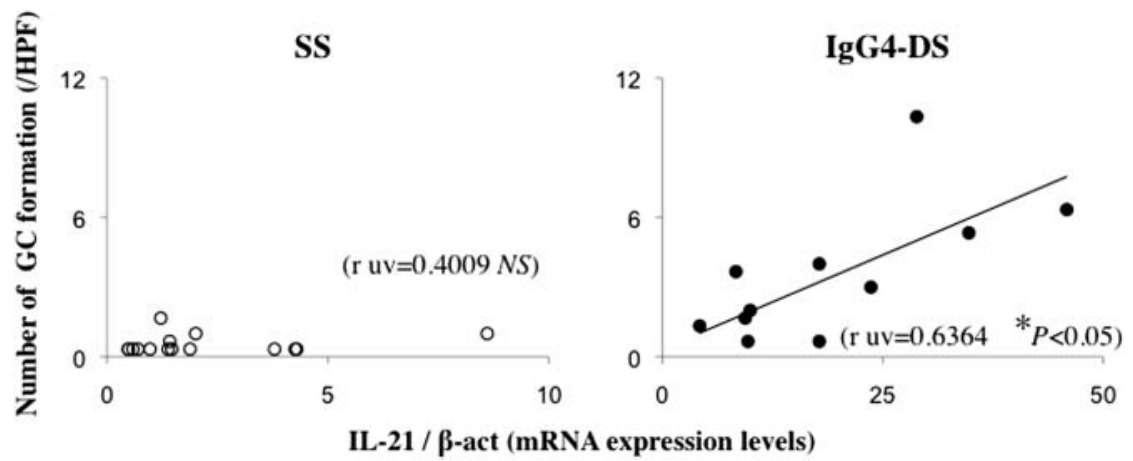

Figure 4 Expression of interleukin 21 (IL-21) may be correlated with ectopic germinal centre (GC) formation in the labial salivary glands (LSGs) of patients with IgG4-related dacryoadenitis and sialoadenitis (IgG4-DS). Correlations between the number of GC formations and IL-21 mRNA expression level in the LSGs from patients with Sjögren's syndrome (SS) $(n=15)$ and those with IgG4-DS $(n=12)$. Real-time PCR products for IL-21 were quantitatively estimated as described in the Methods section. Numbers of GC per high-power field (HPF) were counted in $4 \mathrm{~mm}^{2}$ sections from five different areas as described in the Methods section. Significance of differences between groups was determined by Spearman rank correlation $\left({ }^{*} \mathrm{p}<0.05\right)$. NS, not significant.

selective infiltration of IgG4+ plasma cells around the acini and ductal cells and only mild destruction of the acini compared with patients with SS (figure 5B). Furthermore, the level of IL-21 mRNA expression in LSGs from patients with IgG4-DS was correlated with the IgG4/IgG ratio in immunohistochemically positive cells (figure $5 \mathrm{C}$ ).

\section{DISCUSSION}

In 1953, Morgan and Castleman examined specimens from 18 cases diagnosed with $\mathrm{MD} .{ }^{36}$ Because of the histological similarities between $\mathrm{SS}$ and $\mathrm{MD}$, they reported that almost all cases diagnosed with $\mathrm{MD}$ were considered to be SS. ${ }^{37}$ However, the results of the present study show that the pattern of GC formation in LSGs from patients with SS and $\mathrm{MD}$ (recently named IgG4-DS) were distinctly different; $\mathrm{MD}$ has more hyperplastic GCs than SS. We therefore focused on the mechanism of multiple GC formation in IgG4-DS.

IL-21 has been reported to control the functional activity of effector Th cells and to promote ectopic GC formation by Tfh cells, which were recently shown to contribute to impaired B cell differentiation. ${ }^{17}$ In mice responding to helminth antigens, Tfh cells differentiating from Th2 cells help GC formation in the lymph nodes. We therefore examined the expression of IL-21 and Tfh-related molecules (CXCR5 and Bcl-6) in LSGs from SS and IgG4-DS patients. Immunohistochemical staining indicated that CXCR5 expression was strongly detected in ectopic GCs from both SS and IgG4-DS patients. Interestingly, IL-21 and Bcl-6 expression was strongly detected outside ectopic GCs only in patients with IgG4-DS. As noted above, IL-21 is mainly produced by Th2 and Th17 cells in addition to Tfh cells. ${ }^{15-18} 38$ In order to reveal the differences in IL-21-expressing infiltrating lymphocytes in LSGs between SS and IgG4-DS, we also analysed the expression of Th2-related molecules (IL-4, CCR4 and c-Maf) and Th17-related molecules (IL-17 and RORC2). The expression patterns of Th2-related molecules in LSGs were similar to that of IL-21 in patients with IgG4-DS. In contrast, Th17-related molecules were rarely expressed in patients with IgG4-DS. In this study the mRNA expression levels of IL-21 and IL-17 in the LSGs from patients with SS were higher than those in controls. Furthermore, the expression of IL-21 was detected more strongly in and around the ectopic GC than the ductal epithelial cells in patients with SS. A recent study reported that IL-21 alone was capable of directly inducing both B lymphocyte-induced maturation protein-1 (Blimp-1), which is required for plasma cell differentiation, and Bcl-6, which is required for GC formation. ${ }^{18}$ Hiramatsu et $a l^{39}$ recently reported that c-Maf, which is a Th2 cell-specific transcription factor that activates the IL-4 promoter, directly induced IL-21 production in CD4 $\mathrm{T}$ cells. Furthermore, in the present study we found that IL-21 was positively correlated with the number of GC formations in LSGs from patients with IgG4-DS. These findings suggest that excessive IL-21 production by Th2 cells in salivary glands from patients with IgG4-DS might induce expression of Bcl-6 in B cells resulting in multiple GC formations.

On the other hand, several studies have reported that IL-21 and IL-17 were detected in epithelial and infiltrating mononuclear cells in LSGs from patients with SS and may play an important role in the pathogenesis of SS. ${ }^{40-42}$ These results are consistent with our present data. In this study the mRNA expression levels of IL-21 and IL-17 in LSGs from patients with SS were higher than those from controls. Furthermore, the expression of IL-21 was detected more strongly in and around the ectopic GC than the ductal epithelial cells of patients with SS. In contrast, the expression of IL-17 was detected more strongly in and around the ductal epithelial cells than the ectopic GC from patients with SS. In addition, our previous study indicated that mRNA expression levels of Th17-related molecules in the LSGs from patients with SS were significantly higher than in controls. ${ }^{21}$

Allergic immune responses are known to be caused by allergen-specific Th2-type cytokines IL-4 and IL-13 which are responsible for IgG4 and IgE induction by B cells. ${ }^{43} \mathrm{~A}$ previous study indicated that IL-10 decreased IL-4-induced IgE switching but increased IL-4-induced IgG4 production. $^{44}$ We previously reported that class switching of IgG4 in IgG4-DS is caused by IL-4 and IL-10. ${ }^{21}$ Furthermore, several studies have reported that IL-21 directly inhibits IL-4-induced IgE production, ${ }^{24}$ and class switching of IgG4 is caused by co-stimulation with IL-4 and IL-21 in humans and mice. ${ }^{23}$ In addition, IL-21 induced IL-10 production by mitogen-stimulated peripheral blood mononuclear cells in humans. ${ }^{25}$ We therefore suggested that IL-21 correlated with IL-4 and IL-10 in the class switching of IgG4. In the current study we found that IL-21 was positively correlated with the IgG4/IgG mRNA expression ratio by real-time PCR analyses and the $\operatorname{IgG} 4 / \operatorname{IgG}$ ratio in immunohistochemicallypositive cells. These results suggest that IL-21 might also be involved in the class switching of IgG4 in IgG4-DS. However, 
A

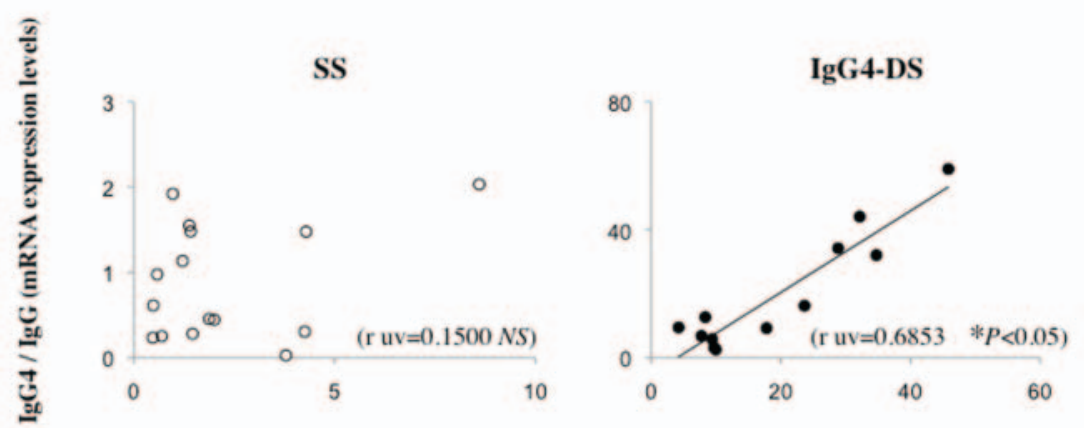

IL-21 / $\beta$-act (mRNA expression levels)

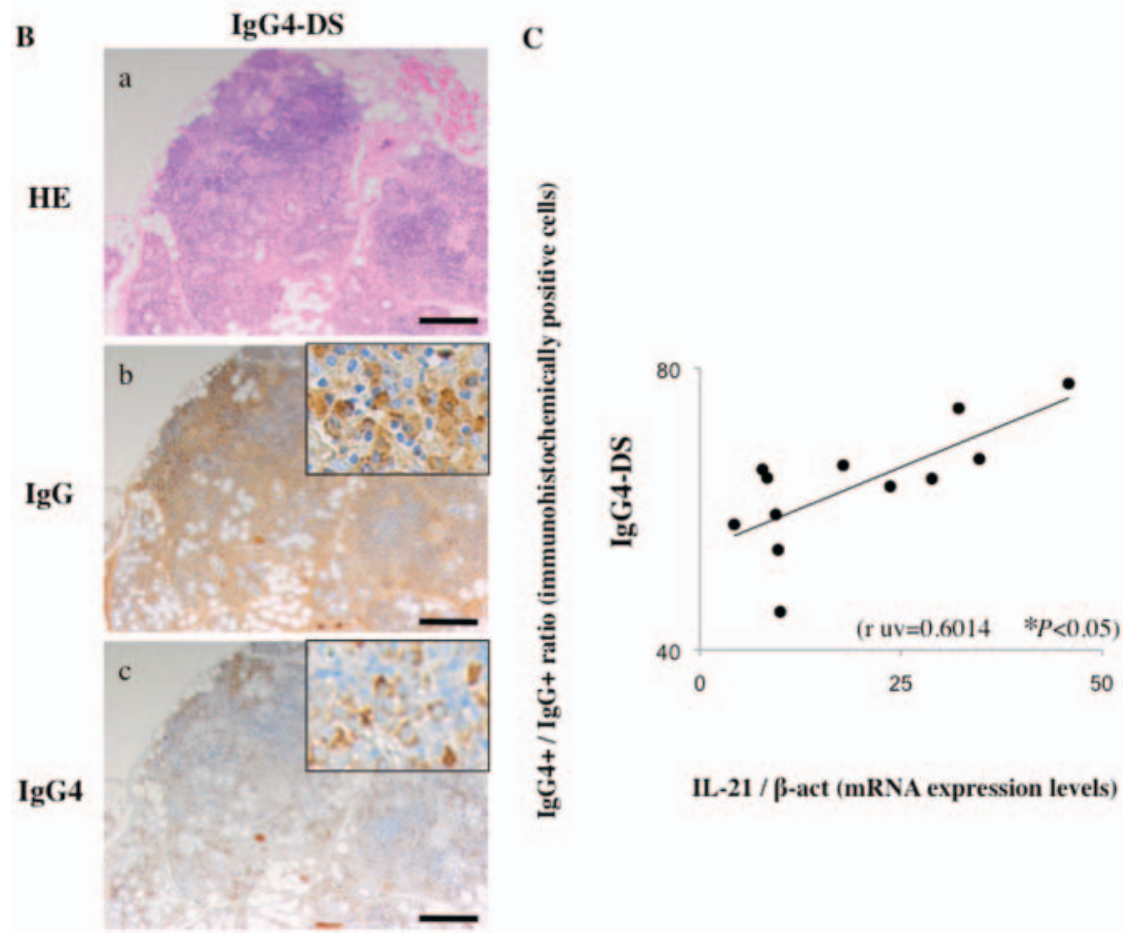

Figure 5 lgG4 production may be correlated with expression of interleukin 21 (IL-21) in the labial salivary glands (LSGs) of patients with IgG4-related dacryoadenitis and sialoadenitis (IgG4-DS). (A) Correlations between the IgG4/lgG mRNA expression ratio and IL-21 mRNA expression level in the LSGs from patients with Sjögren's syndrome (SS) $(n=15)$ and those with IgG4-DS $(n=12)$. Real-time PCR products for IL-21, IgG and IgG4 were quantitatively estimated as described in the Methods section. (B) $\operatorname{lgG} 4+$ cell ratio $(\%)=\lg G 4+$ cells/lgG + cells 100 . Counts were made in $1 \mathrm{~mm}^{2}$ sections from five different areas. (C) Correlations between the frequencies of IgG4+ cells and levels of IL-21 mRNA in the LSGs from patients with IgG4-DS $(n=12)$. Significance of differences between groups was determined by Spearman rank correlation $\left({ }^{*} p<0.05\right)$. NS, not significant.

more case reports and further examinations are required to elucidate the pathogenesis of the disease.

In this study we have confirmed that the overexpression of IL-21 by Th2 cells is involved in the induction of multiple GC formation and IgG4 production in IgG4-DS. However, further studies are needed, including the establishment of a mouse model of IgG4-DS. A more thorough understanding of the complex mechanisms of IgG4-DS, especially the role of Th subset-related cytokines, could lead to the development of novel pharmacological strategies aimed at disrupting the cytokine network and inhibiting the initiation and/or progression of IgG4-DS.

Contributors Study conception and design: TM, MM. Acquisition of data: J-NH, AT, SS, YK. Analysis and interpretation of data: HN, KM, SN

Funding This work was supported in part by grants from the Ministry of Education, Culture, Sports, Science, and Technology of Japan (22791990) and by Intractable
Diseases, Health and Labour Sciences Research Grants from the Ministry of Health, Labour, and Welfare of Japan (H23-005, H24-043).

Competing interest None.

Patient consent Obtained.

Ethics approval Ethics approval was obtained from the ethics committee of Kyushu University, Japan.

Provenance and peer review Not commissioned; externally peer reviewed.

Correction notice This article has been corrected since it was published online first. Changes have been made to figure 2 and figure 3 legends.

\section{REFERENCES}

1. Yamamoto M, Takahashi $\mathrm{H}$, Sugai S, et al. Clinical and pathological characteristics of Mikulicz's disease (IgG4-related plasmacytic exocrinopathy). Autoimmun Rev 2005:4:195-200.

2. Yamamoto $\mathbf{M}$, Takahashi $\mathrm{H}$, Ohara M, et al. A new conceptualization for Mikulicz's disease as an IgG4-related plasmacytic disease. Mod Rheumatol 2006;16:335-40. 
3. Hamano H, Kawa S, Horiuchi $A$, et al. High serum lgG4 concentrations in patients with sclerosing pancreatitis. N Engl J Med 2001;344:732-8.

4. Zen Y, Harada K, Sasaki M, et al. IgG4-related sclerosing cholangitis with and without hepatic inflammatory pseudotumor, and sclerosing pancreatitis-associated sclerosing cholangitis: do they belong to a spectrum of sclerosing pancreatitis? Am J Surg Pathol 2004;28:1193-203.

5. Takeda S, Haratake J, Kasai T, et al. IgG4-associated idiopathic tubulointerstitial nephritis complicating autoimmune pancreatitis. Nephrol Dial Transplant 2004;19:474-6.

6. Zen Y, Kitagawa S, Minato $\mathrm{H}$, et al. IgG4-positive plasma cells in inflammatory pseudotumor (plasma cell granuloma) of the lung. Hum Pathol 2005;36:710-17.

7. Hamed G, Tsushima K, Yasuo M, et al. Inflammatory lesions of the lung submandibular gland, bile duct and prostate in a patient with IgG4-associated multifocal systemic fibrosclerosis. Respirology 2007:12:455-7.

8. Kitagawa S, Zen Y, Harada K, et al. Abundant IgG4-positive plasma cell infiltration characterizes chronic sclerosing sialadenitis (Kuttner's tumor). Am J Surg Pathol 2005;29:783-91

9. Yamamoto $\mathbf{M}$, Takahashi $H$, Naishiro $Y$, et al. Mikulicz's disease and systemic IgG4-related plasmacytic syndrome (SIPS). Nihon Rinsho Men'eki Gakkai Kaishi (Jpn J Clin Immunol) 2008;31:1-8.

10. Masaki Y, Umehara H. IgG4-related disease-the diagnostic confusion and how to avoid it. Nihon Rinsho Men'eki Gakkai Kaishi (Jpn J Clin Immunol) 2009;32:478-83.

11. Umehara H, Okazaki K, Masaki Y, et al. A novel clinical entity, lgG4-related disease (lgG4RD): general concept and details. Mod Rheumatol/Jpn Rheum Assoc 2012:22:1-14.

12. Stone JH, Zen Y, Deshpande V. IgG4-related disease. N Engl J Med 2012;366:539-51.

13. Harrison JD, Rodriguez-Justo M. Commentary on IgG4-related sialadenitis: Mikulicz's disease, Küttner's tumor, and eponymy. Histopathology 2011;58:1164-6.

14. Kakuchi Y, Yamada K, Suzuki Y, et al. IgG4-related skin lesions in a patient with IgG4-related chronic sclerosing dacryoadenitis and sialoadenitis. Intern Med 2011;50:1465-9.

15. Wurster AL, Rodgers VL, Satoskar AR, et al. Interleukin 21 is a Thelper (Th) cell 2 cytokine that specifically inhibits the differentiation of naive Th cells into interferon gamma-producing Th1 cells. J Exp Med 2002;196:969-77.

16. Nurieva R, Yang X0, Martinez G, et al. Essential autocrine regulation by IL-21 in the generation of inflammatory T cells. Nature 2007;448:480-3.

17. Vinuesa CG, Tangye SG, Moser B, et al. Follicular B helper T cells in antibody responses and autoimmunity. Nat Rev Immunol 2005;5:853-65.

18. Ettinger R, Kuchen S, Lipsky PE. Interleukin 21 as a target of intervention in autoimmune disease. Ann Rheum Dis 2008;67:iii83-6.

19. Miyake K, Moriyama M, Aizawa K, et al. Peripheral CD4+ T cells showing a Th2 phenotype in a patient with Mikulicz's disease associated with lymphadenopathy and pleural effusion. Mod Rheumatol 2008;18:86-90.

20. Nakashima H, Miyake K, Moriyama M, et al. An amplification of IL-10 and TGF-beta in patients with IgG4-related tubulointerstitial nephritis. Clin Nephrol 2010;73:385-91.

21. Tanaka A, Moriyama $\mathrm{M}$, Nakashima $\mathrm{H}$, et al. Th2 and regulatory immune reactions contributes to IgG4 production and the initiation of Mikulicz's disease. Arthritis Rheum 2012;64:254-63.

22. Ray K. Connective tissue disease: immune responses in Mikulicz disease differ from those in SS. Nat Rev Rheumatol 2011;7:621.

23. Kitayama D, Sakamoto A, Arima $M$, et al. A role for Bcl6 in sequential class switch recombination to $\lg E$ in B cells stimulated with IL-4 and IL-21. Mol Immunol 2008;45:1337-45.

24. Suto A, Nakajima $H$, Hirose $K$, et al. Interleukin 21 prevents antigen-induced lgE production by inhibiting germ line $\mathrm{C}$ (epsilon) transcription of IL-4-stimulated $\mathrm{B}$ cells. Blood 2002;100:4565-73.
25. Wood N, Bourque K, Donaldson DD, et al. IL-21 effects on human IgE production in response to IL-4 or IL-13. Cell Immunol 2004;231:133-45.

26. Vitali C, Bombardieri S, Jonsson R, et al. Classification criteria for Sjogren's syndrome: a revised version of the European criteria proposed by the American-European Consensus Group. Ann Rheum Dis 2002;61:554-8.

27. Greenspan JS, Daniels TE, Talal N, et al. The histopathology of Sjogren's syndrome in labial salivary gland biopsies. Oral Surg Oral Med Oral Pathol 1974;37:217-29

28. Daniels TE, Whitcher JP. Association of patterns of labial salivary gland inflammation with keratoconjunctivitis sicca. Analysis of 618 patients with suspected Sjogren's syndrome. Arthritis Rheum 1994;37:869-77.

29. Szodoray P, Alex P, Jonsson MV, et al. Distinct profiles of Sjogren's syndrome patients with ectopic salivary gland germinal centers revealed by serum cytokines and BAFF Clin Immunol 2005:117:168-76.

30. Salomonsson S, Jonsson MV, Skarstein K, et al. Cellular basis of ectopic germinal center formation and autoantibody production in the target organ of patients with Sjogren's syndrome. Arthritis Rheum 2003;48:3187-201.

31. Takemura S, Braun A, Crowson $C$, et al. Lymphoid neogenesis in rheumatoid synovitis. J Immunol (Baltimore) 2001;167:1072-80.

32. Sasaki M, Nakamura S, Ohyama $Y$, et al. Accumulation of common T cell clonotypes in the salivary glands of patients with human T lymphotropic virus type I-associated and idiopathic Sjogren's syndrome. J Immunol (Baltimore) 2000;164:2823-31.

33. Acosta-Rodriguez EV, Napolitani G, Lanzavecchia A, et al. Interleukins 1 beta and 6 but not transforming growth factor-beta are essential for the differentiation of interleukin 17-producing human T helper cells. Nat Immunol 2007;8:942-9.

34. Ohyama Y, Nakamura S, Matsuzaki G, et al. Cytokine messenger RNA expression in the labial salivary glands of patients with Sjogren's syndrome. Arthritis Rheum 1996;39:1376-84.

35. Hiroki A, Nakamura S, Shinohara $\mathrm{M}$, et al. A comparison of glandular involvement between chronic graft-versus-host disease and Sjogren's syndrome. Int J Oral Maxillofacial Surg 1996;25:298-307.

36. Morgan WS, Castleman B. A clinicopathologic study of Mikulicz's disease. Am J Pathol 1953:29:471-503.

37. Morgan WS. The probable systemic nature of Mikulicz's disease and its relation to Sjogren's syndrome. N Engl JMed 1954;251:5-10.

38. Crotty S. Follicular helper CD4T cells (TFH). Annu Rev Immunol 2011;29:621-63.

39. Hiramatsu $\mathbf{Y}$, Suto A, Kashiwakuma D, et al. c-Maf activates the promoter and enhancer of the IL-21 gene, and TGF-beta inhibits c-Maf-induced IL-21 production in CD4+ T cells. J Leukoc Biol 2010;87:703-12

40. Kang KY, Kim HO, Kwok SK, et al. Impact of interleukin-21 in the pathogenesis of primary Sjögren's syndrome: increased serum levels of interleukin-21 and its expression in the labial salivary glands. Arthritis Res Ther 2011;13:R179.

41. Katsifls GE, Rekka S, Moutsopoulos NM, et al. Systemic and local interleukin-17 and linked cytokines associated with Sjögren's syndrome immunopathogenesis. Am J Pathol 2009;175:1167-77.

42. Sakai A, Sugawara Y, Kuroishi T, et al. Identification of IL-18 and Th17 cells in salivary glands of patients with Sjögren's syndrome, and amplification of IL-17-mediated secretion of inflammatory cytokines from salivary gland cells by IL-18. J Immunol 2008;181:2898-906.

43. Finkelman FD, Vercelli D. Advances in asthma, allergy mechanisms, and genetics in 2006. J Allergy Clin Immunol 2007;120:544-50.

44. Meiler $\mathbf{F}$, Klunker S, Zimmermann $\mathrm{M}$, et al. Distinct regulation of $\lg \mathrm{E}$ lgG4 and IgA by T regulatory cells and toll-like receptors. Allergy 2008;63:1455-63. 